\title{
Restabelecimento de Energia com Busca Local na Região de Falta e Possibilidade de Corte de Cargas
}

\author{
José Paulo R. Fernandes*, Etiane O. P. de Carvalho*, Leandro T. Marques**, João Bosco A. London Jr.* \\ *Escola de Engenharia de São Carlos, Universidade de São Paulo, São Carlos - SP, 13566-590 \\ Brasil (e-mail: joseprf@usp.br, etiane@usp.br, jbalj@sc.usp.br). \\ **Instituto de Ciências Tecnológicas e Exatas, Universidade Federal do Triangulo Mineiro, Uberaba - MG, 38064-200 \\ Brasil (e-mail: leandrotolomeu@gmail.com).
}

\begin{abstract}
Solutions to the service restoration problem are extremely important to distribution systems' operators. Recently a practical and efficient Multi-Objective Evolutionary Algorithm (MOEA) in subpopulation tables was developed to generate power restoration plans. It was demonstrated through tests performed on the real and large-scale distribution system of Londrina City, Brazil. This paper proposes an extension of this MOEA that incorporates a search procedure applied to the fault region neighborhood. Tests performed on a distribution system presented in the literature indicate that, in relation to that MOEA in tables, the extended methodology can improve solutions diversity and have a better performance in terms of the problem objectives of minimizing energy not supplied and switching operations.
\end{abstract}

Resumo: Soluções para o problema de restabelecimento de energia são de extrema importância para as empresas distribuidoras de energia. Recentemente foi desenvolvido um Algoritmo Evolutivo MultiObjetivo (AEMO) em Tabelas que possibilita a obtenção de planos de restabelecimento de energia mesmo para sistemas de grande porte em intervalos de tempo factíveis para aplicação em tempo real. Destaca-se que essa metodologia foi validada no sistema de distribuição real de grande porte da cidade de Londrina, Brasil. Este artigo propõe uma extensão desse AEMO, a partir da incorporação de uma busca determinística na vizinhança da região de falta, para obtenção de novas soluções. Testes realizados em um sistema da literatura indicam que, em relação ao AEMO utilizado como base, a metodologia estendida consegue aumentar a diversidade das soluções encontradas e melhorar o desempenho em relação aos objetivos de minimizar a energia não suprida e os chaveamentos.

Keywords: Service Restoration; Multi-Objective Evolutionary Algorithm; Load Shedding; Local Search; Distribution System.

Palavras-chaves: Restabelecimento de Energia; Algoritmo Evolutivo Multi-objetivo; Corte de Carga; Busca local; Sistemas de Distribuição.

\section{INTRODUÇÃO}

O sistema elétrico de potência (SEP) está sujeito a interrupções no fornecimento de energia, ocasionadas por fenômenos da natureza, acidentes de trânsito, falhas em equipamentos de energia, dentre outros, causando prejuízos aos consumidores e às concessionárias de energia. Para garantir que os SEPs funcionem de maneira segura e contínua, foram criadas normas para regulamentar seu funcionamento, e o descumprimento dessas pode resultar em punições às prestadoras de serviços elétricos. Dentre os principais indicadores de qualidade de serviço estabelecidos pela Agência Nacional de Energia Elétrica - ANEEL (2018), estão a Duração Equivalente de Interrupção por Unidade Consumidora (DEC) e a Frequência Equivalente de Interrupção por Unidade Consumidora (FEC), computados para as interrupções no fornecimento de energia de duração maior que 3 minutos.

Neste contexto, para garantir que as concessionárias consigam melhorar seus indicadores de continuidade de energia, o fornecimento de soluções de qualidade para o problema de restabelecimento de energia é de extrema importância. Tratase de um problema complexo e desafiador, pois leva em consideração diversos fatores e variáveis. Um exemplo disto está no conflito entre os seus principais objetivos, que visam, simultaneamente, a redução de consumidores desligados e do número de manobras em chaves. Desse modo, os elevados prejuízos gerados pelas interrupções de energia e o desenvolvimento de novas tecnologias e métodos de otimização justificam o avanço de pesquisas destinadas à obtenção computacional de Planos de Restabelecimento de Energia (PREs).

A metodologia proposta neste trabalho consiste na adição de funcionalidades que buscam aprimorar o desempenho do Algoritmo Evolutivo Multi-objetivo em Tabelas (AEMT) apresentado em (Marques 2018). Esse método base é capaz de lidar com sistemas de grande porte sem simplificações, dar preferência a consumidores prioritários e chaves controladas remotamente e avaliar e fornecer uma sequência de chaveamento. As funções propostas neste artigo consistem em: 
buscar por PREs capazes de evitar desligamentos temporários desnecessários e indesejáveis; e cortar cargas não prioritárias inicialmente não afetadas pela falta quando isto for necessário para o atendimento de cargas prioritárias desligadas. Resultados de simulações computacionais demonstram que essas funções possibilitam uma ampliação da varredura do espaço de busca, por explorar soluções que incluem manobras de corte antes desconsideradas.

\section{ENUNCIADO FORMAL DO PROBLEMA}

Os valores de Energia Não-Suprida (ENS) permitem avaliar a relação entre a potência restaurada e o tempo necessário para realizar as manobras em chaves seccionadoras, sendo mais impactante nos indicadores de continuidade de energia do que o número final de consumidores desligados ou a Potência NãoSuprida (PNS), como proposto por Mohammadi e Afrakhteh (2012). Assim, o problema de restabelecimento de energia pode ser formalizado como um problema multi-objetivo com a finalidade de minimizar a ENS e o número de manobras, levando em conta as restrições de operação do sistema (Shin et al. 2004). Considerando então a necessidade de definição de uma sequência adequada de chaveamento e a representação de Sistemas de Distribuição (SDs) através de grafos, formalmente pode-se definir o problema de restabelecimento de energia em SDs da seguinte forma (Marques 2018):

$$
\begin{array}{r}
\text { Min. } \varepsilon(G), \psi(G) \\
\text { s.a: } A\left(G^{e}\right) x\left(G^{e}\right)=c\left(G^{e}\right) \\
A\left(G^{e}\right) Y_{x}\left(G^{e}\right) A\left(G^{e}\right)^{t} v\left(G^{e}\right)=c\left(G^{e}\right) \\
X\left(G^{e}\right) \leq 1 \\
B\left(G^{e}\right) \leq 1 \\
V\left(G^{e}\right) \leq \delta \\
G \text { deve ser radial } \\
G=G^{e} \cup G^{\text {ne }} \\
\text { seq }(G) \text { ser factivel }
\end{array}
$$

onde, G é uma configuração radial do SD representado por uma floresta de grafos, $G^{\text {e }}$ é a porção da rede que se encontra em serviço, $G^{\text {ne }}$ é a porção da rede que se encontra fora de serviço, $\varepsilon(\mathrm{G})$ é a energia total não suprida durante o intervalo de tempo necessário para a execução das manobras para obtenção de $\mathrm{G}$ a partir de $\mathrm{G}^{1}$, a qual consiste na configuração da rede na qual os setores em falta foram identificados e isolados (um setor é um agrupamento de barras separado por chaves) - logo, na configuração $G^{1}$, tendo em vista a característica radial de operação dos $\mathrm{SD}$, os setores saudáveis a jusante dos setores em falta estarão também desligados, $\psi(\mathrm{G})$ é o número total de manobras para obtenção de $\mathrm{G}$ a partir da $\mathrm{G}^{1}, \mathrm{~A}\left(\mathrm{G}^{\mathrm{e}}\right)$ é a matriz incidência nó-aresta pseudo-orientada (obtida através de um grafo orientado das correntes) de $G^{\mathrm{e}}$, $\mathrm{x}\left(\mathrm{G}^{\mathrm{e}}\right)$ é o vetor de correntes complexas nas linhas em $\left(\mathrm{G}^{\mathrm{e}}\right)$, $\mathrm{c}\left(\mathrm{G}^{\mathrm{e}}\right)$ é o vetor de correntes complexas demandadas em barras de carga ou injetadas em barras de subestações em $\left(G^{e}\right), Y_{x}\left(G^{e}\right)$ é a matriz diagonal de admitâncias da rede em $\left(G^{e}\right), v\left(G^{e}\right)$ é o vetor de tensões complexas nas barras em $(\mathrm{Ge}), \mathrm{X}(\mathrm{G})$ é o máximo valor de carregamento de rede em $(\mathrm{G})$, dado por $\mathrm{X}(\mathrm{G})=\operatorname{MAX}\left\{x_{j} / \bar{x}_{\jmath}\right\}$, no qual $\bar{x}_{\jmath}$ é um limitante superior de corrente para cada magnitude de corrente $x_{j}$ em uma linha $\mathrm{j}$, $\mathrm{B}(\mathrm{G})$ é o máximo valor para carregamento de subestação (transformador) em $\mathrm{G}$, dado por $\mathrm{B}(\mathrm{G})=\operatorname{MAX}\left\{b_{s} / \bar{b}_{s}\right\}$, sendo $\bar{b}_{s}$ um limitante superior para cada magnitude de injeção de corrente bs provida por uma subestação $\mathrm{s}, \mathrm{V}(\mathrm{G})$ é a máxima queda de tensão em $\mathrm{G}$, dada por $\mathrm{V}(\mathrm{G})=M A X\left\{\left|v_{s}-v_{k}\right| / \delta\right\}$, sendo $v_{s}$ a magnitude de tensão na barra de uma subestação $\mathrm{s}$ (mais especificamente, na barra secundária do transformador da subestação s), $v_{k}$ é a magnitude de tensão em uma barra $\mathrm{k}$ da rede e $\delta$ é a máxima queda de tensão admissível, seq $(G)$ é a sequência de chaveamento necessária para sair de G1 e chegar em $G$, e é dita factível sempre que as configurações intermediárias (obtidas entre $\mathrm{G}^{1}$ e $\mathrm{G}$ ) estão dentro de um limite relaxado (podendo ultrapassar o máximo carregamento de subestação e a máxima queda de tensão por margens pequenas pré-definidas) e a configuração final $\mathrm{G}$ atende à todas as restrições do problema.

Outro aspecto importante para modelagem de SDs neste trabalho é o uso da Representação Nó-Profundidade (RNP), que consiste numa estrutura de dados computacionalmente eficiente, proposta por Delbem et al. (2004), para o armazenamento de árvores de grafo. Neste trabalho são propostos dois tipos de RNPs: um para representar as porções energizadas da rede em estudo e outro para as porções desenergizadas restauráveis da rede. Ademais, ela possui operadores que garantem a geração de configurações radiais, assegurando, assim, que essa restrição do problema seja sempre atendida.

\section{METODOLOGIA}

\subsection{Método base}

Os AEMTs trabalham em paralelo com várias subpopulações armazenadas em tabelas, onde as melhores soluções (ou configurações do SD), para cada característica do problema (objetivos e restrições técnicas), são armazenadas em suas respectivas tabelas (Benayoun et al. 1971). Para exemplificar o funcionamento de um AEMT, suponha o tratamento do problema de restabelecimento com os dois objetivos citados anteriormente, sujeito às restrições de carregamento de rede $\mathrm{e}$ subestação e queda de tensão. Poderá existir, então, cinco tabelas, uma referente a cada objetivo ou restrição. Assim, os melhores indivíduos para um objetivo ou restrição estarão na tabela associada a este objetivo ou restrição. Podem existir também tabelas estabelecendo compromissos entre os extremos mínimos e/ou máximos dos dois objetivos do problema. $\mathrm{O}$ uso de tabelas também permite que o operador do sistema possa escolher buscar por soluções com diferentes características. Estas características podem incluir objetivos importantes ao SD, como perdas reduzidas, baixa queda de tensão e menor carregamento de rede.

O método proposto consiste no aperfeiçoamento do método apresentado por Marques (2018), que consiste numa combinação do Algoritmo Evolutivo Multi-objetivo em Tabelas (AEMT) proposto por Santos et al. (2010) com alguns princípios do Nondominated Sorting Genetic Algorithm-II 
(NSGA-II) (Deb et al. 2002), a fim de lidar com vários aspectos práticos do problema.

Várias etapas compõem o método base, partindo do isolamento do setor em falta até o fornecimento de PREs para o operador, que incluem uma sequência de chaveamento. $\mathrm{O}$ método pode ser dividido, de modo simplificado, em seis grandes etapas: leitura de dados, obtenção da configuração $G^{1}$, que é o indivíduo inicial (indivíduo no qual todos os setores saudáveis afetados estão desligados e o setor em falta está isolado), execução de uma Busca Exaustiva Local Modificada (BELM) (modificada, porque na verdade é uma extensão da busca determinística proposta em (Camillo et al. 2016)), geração da população inicial, processo evolutivo e fornecimento das soluções.

Na etapa de execução do processo evolutivo é utilizado o princípio de tabelas de subpopulação, segundo o qual, a cada geração, a população é dividida em subpopulações e armazenadas em tabelas, as quais representam alguma característica de interesse. Este princípio, e os diversos AEMOs que o aplicam, tem a vantagem de tratar vários objetivos simultaneamente, sem a necessidade da utilização de critérios de penalização ou ponderação.

Esse método ainda tem como vantagem a utilização da RNP, para a representação do SD, uso dos operadores de reprodução que garantem que a restrição de manutenção da radialidade seja sempre atendida, realização de fluxo de potência computacionalmente eficiente por varredura direta-inversa, capacidade para lidar com SDs de grande porte, priorização de chaves remotas e carga prioritárias e verificação de sequência de chaveamento (Marques 2018).

\subsection{Método proposto}

A metodologia proposta adiciona, ao método apresentado por Marques (2018), duas novas funções: uma busca local que expande a BELM e o operador de corte de carga, modificando o comportamento ao longo do processo evolutivo. Preservando a estrutura consolidada do método base, manteve-se o uso da RNP, seus operadores, bem como, a leitura de dados, parâmetros de entrada, número de tabelas de subpopulação e critérios de seleção dos PREs a serem apresentados ao operador.

Inicialmente, a BELM testa o religamento de cargas, em setores sãos que foram desligados, somente com as chaves normalmente abertas (NAs) da primeira vizinhança (chaves que conectam algum dos setores sãos que foram desligados a setores energizados). Quando a BELM não encontra uma solução factível, realiza-se a Busca Local para avaliar a factibilidade das configurações possíveis de serem obtidas operando somente chaves NAs e chaves normalmente fechadas (NFs) da primeira vizinhança dos setores sãos que foram desligados que conectem tais setores a setores energizados. Isso significa que, além de testar mais possibilidades de restauração que a BELM, serão gerados novos indivíduos que podem ser utilizados no processo evolutivo.
Outra contribuição do método proposto é o operador de corte de carga, que é incluso no processo evolutivo e permite a realização de manobras de corte de carga em alimentadores que não sofreram desligamento em função da ocorrência e isolação da falta. Assim, a exploração do espaço de busca irá abranger indivíduos com setores cortados (desenergizados) que não foram inicialmente desligados pelo isolamento dos setores em falta. O fluxograma do método proposto, apresentado na Fig. 1, mostra apenas as principais etapas da metodologia proposta.

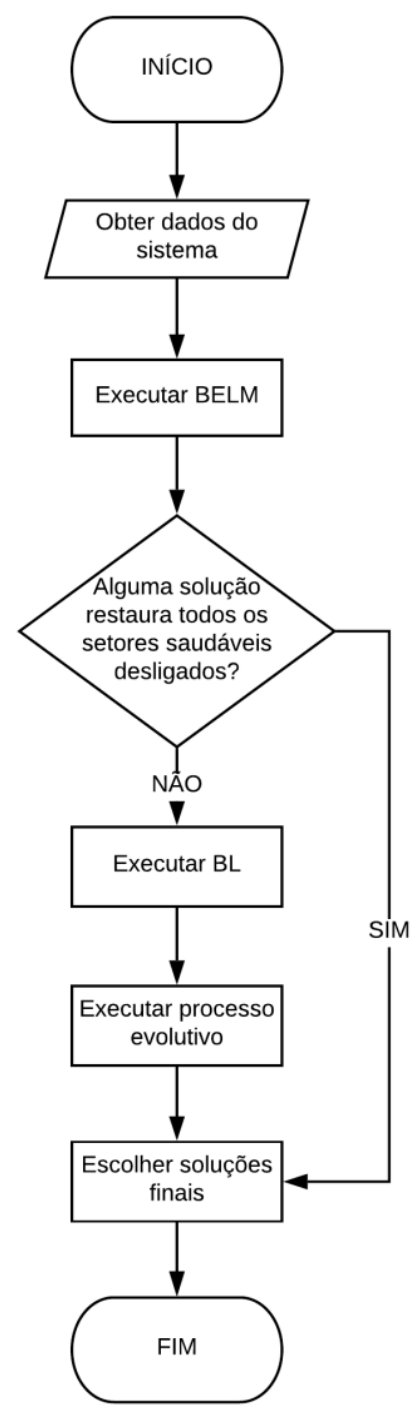

Fig. 1 Fluxograma simplificado mostrando as principais etapas do método proposto.

\subsubsection{Busca Local}

Define-se, como Busca Local (BL), a busca proposta neste trabalho executada após a BELM. No método base, a BELM é executada previamente ao AEMT. A BELM não é excluída do método base, pois requer menor esforço computacional, caso seja encontrado uma solução factível com número mínimo de manobras necessárias restaurando todos os setores saudáveis desligados, uma vez que, ao encontrar essa solução o programa é finalizado. 
Consideram-se, na BL, todas as chaves NAs que ligam um setor energizado a um sadio desenergizado restaurável (que pode ser religado enquanto a causa da falta é resolvida) e as chaves NFs que conectam setores sadios desenergizados restauráveis entre si, chamadas de chaves do Tier 1 (Miu, Chiang e Mcnulty 2000), ou seja, explora as combinações possíveis focando somente na região problema. A consideração das chaves NFs causa um aumento no número de combinações possíveis, exigindo uso de artifícios para reduzir o uso de memória e o tempo de execução do método por meio de filtros que avaliam quais combinações devem ser analisadas e organiza a sequência de chaveamento a ser aplicada.

O ponto de partida para os indivíduos gerados nessa busca é a configuração $\mathrm{G}^{1}$, também chamado de indivíduo inicial, citado anteriormente na seção 2 . O método proposto irá testar todas as configurações finais possíveis, entre as chaves presentes no Tier 1, durante a BL. Os indivíduos obtidos nesse processo irão preencher as tabelas de subpopulações utilizadas na etapa evolutiva, ampliando, dessa forma, a região percorrida no espaço de busca, podendo servir também de solução final do método proposto, caso não sejam encontradas soluções melhores.

O primeiro passo desse processo é listar as chaves disponíveis para a busca, ordenando-as de modo que as NAs sejam as primeiras, seguidas pelas chaves NFs. Com todas as chaves organizadas é criado um vetor de estados, onde 0 indica aberta e 1 fechada. Assim, supondo um caso no qual tenha-se duas chaves NAs e três chaves NFs, será criado o vetor [NA1 NA2 NF1 NF2 NF3], que pode ser tratado com um número binário, cuja contagem é bastante simples.

Através desse vetor, são geradas todas as combinações de estados de chaves a serem testadas. Essas configurações não são geradas na forma de RNP, mas sim obtidas pela análise dos caminhos formados pelas chaves que se encontram fechadas no vetor de estados. Apesar das vantagens da RNP para lidar com o problema de restabelecimento, ao garantir a radialidade das configurações geradas, seus operadores (Load Reconnect Operador (LRO), Preserve Ancestor Operator (PAO) e o Change Ancestor Operator (CAO)) não são simples de manipular a fim de realizar uma busca exaustiva ordenada na região de falta, ao contrário de um valor binário, conforme utilizado na estrutura proposta. Essa estrutura, então, é convertida para trincas de nós necessárias às aplicações dos operadores da RNP adaptados para trabalhar com dados prédefinidos e nas regiões desenergizadas. A sequência de chaveamento é definida de modo que nenhum consumidor seja ligado ou desligado temporariamente, favorecendo a factibilidade dos indivíduos intermediários, e de modo que seja sempre realizado o mínimo de manobras possível. Porém não há garantia que a ordem escolhida seja a de menor ENS.

A primeira combinação lida na BL será a configuração representante do indivíduo inicial (com o setor em falta isolado, sem nenhum setor reconectado ainda e sem manobras envolvendo os setores saudáveis desenergizados). Considerando novamente o exemplo dado anteriormente, essa configuração seria representada pelo vetor [ $\left[\begin{array}{lllll}0 & 0 & 1 & 1 & 1\end{array}\right]$. Para gerar as combinações seguintes, realiza-se uma contagem no sentido crescente de números binários, ou seja, transformando a primeira combinação em um valor binário, incrementando-a e voltando para a forma vetorial. Deste modo, a segunda configuração do exemplo seria representada pelo vetor [0 10 0 0], indicando que a chave NA2 está fechada e todas as demais abertas, a terceira seria representada por [ $\left[\begin{array}{lllll}0 & 1 & 0 & 0 & 1\end{array}\right]$, com as chaves NA2 e NF3 fechadas e as demais abertas, e assim por diante.

A operação radial da rede é uma restrição operacional, portanto, as combinações obtidas pela BL que formem anéis devem ser ignoradas. Anéis só ocorrem em casos em que haja mais de uma chave NA no vetor de combinações sendo fechadas na mesma configuração. Esta verificação não é necessária quando se trabalha apenas com RNPs, sendo feita apenas na etapa da BL. Além disso, quando há formação de caminhos restritos à região desenergizada, o próprio método de construção da topologia da rede a partir dos elementos do vetor de combinação já ignora essas manobras.

Para que seja possível descartar as combinações que se encaixem nas condições especificadas, um filtro analisa quais são as combinações válidas e quais podem ser ignoradas por desrespeitar a restrição de radialidade ou por terem caminhos desenergizados, sem a necessidade de percorrer todas elas. Para percorrer todas as combinações, sem nenhum filtro e sem tomar como ponto de partida $\mathrm{G}^{1}$, seria necessária a verificação de $2^{\mathrm{n}}$ combinações, onde $\mathrm{n}$ é o número total de chaves no vetor, tornando inviável a aplicação da BL dependendo do valor de n.

As combinações passam por processos de verificação que identificam se em algum momento houve a conexão entre dois setores previamente energizados e se, após a formação de todos os caminhos gerados pelas chaves que se encontram fechadas, há algum caminho que não energiza setor algum. Qualquer um desses dois casos fará a combinação ser ignorada e o método seguirá para a próxima combinação.

Para reduzir o consumo de tempo e recursos computacionais, o filtro, ao identificar que uma combinação é inválida, descobrirá o motivo disso acontecer, observando qual chave é responsável pelo problema, e escolherá a estratégia para obtenção da próxima configuração potencialmente válida. Assim, pelo exemplo utilizado anteriormente, se na

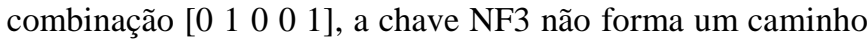
energizado pelo fechamento de NA2, esta combinação será descartada e o filtro irá buscar a próxima combinação que forme um caminho energizado. Similarmente, se as chaves NA1 e NA2 estiverem fechadas (combinações [ $111 \mathrm{X} X \mathrm{X}$ ], X podendo ser 0 ou 1) e formarem um anel qualquer, essa combinação será descartada e o filtro buscará a próxima combinação que não forma anéis.

Por meio da aplicação do filtro, garante-se que não serão gerados indivíduos que diferem entre si apenas pela topologia da região desenergizada e o número de combinações testadas será reduzido.

Ao identificar que não existe mais combinações válidas a serem testadas, o que pode ocorrer pelo fato de a contagem binária ter chegado ao seu valor máximo (todas as posições do 
vetor de combinações iguais a 1) ou pelo fato de não ser mais possível evitar anéis ou obter configurações com diferenças na parte energizada, a BL termina e o método segue para a etapa evolutiva. O fluxograma que representa a BL é mostrado na Fig. 2.

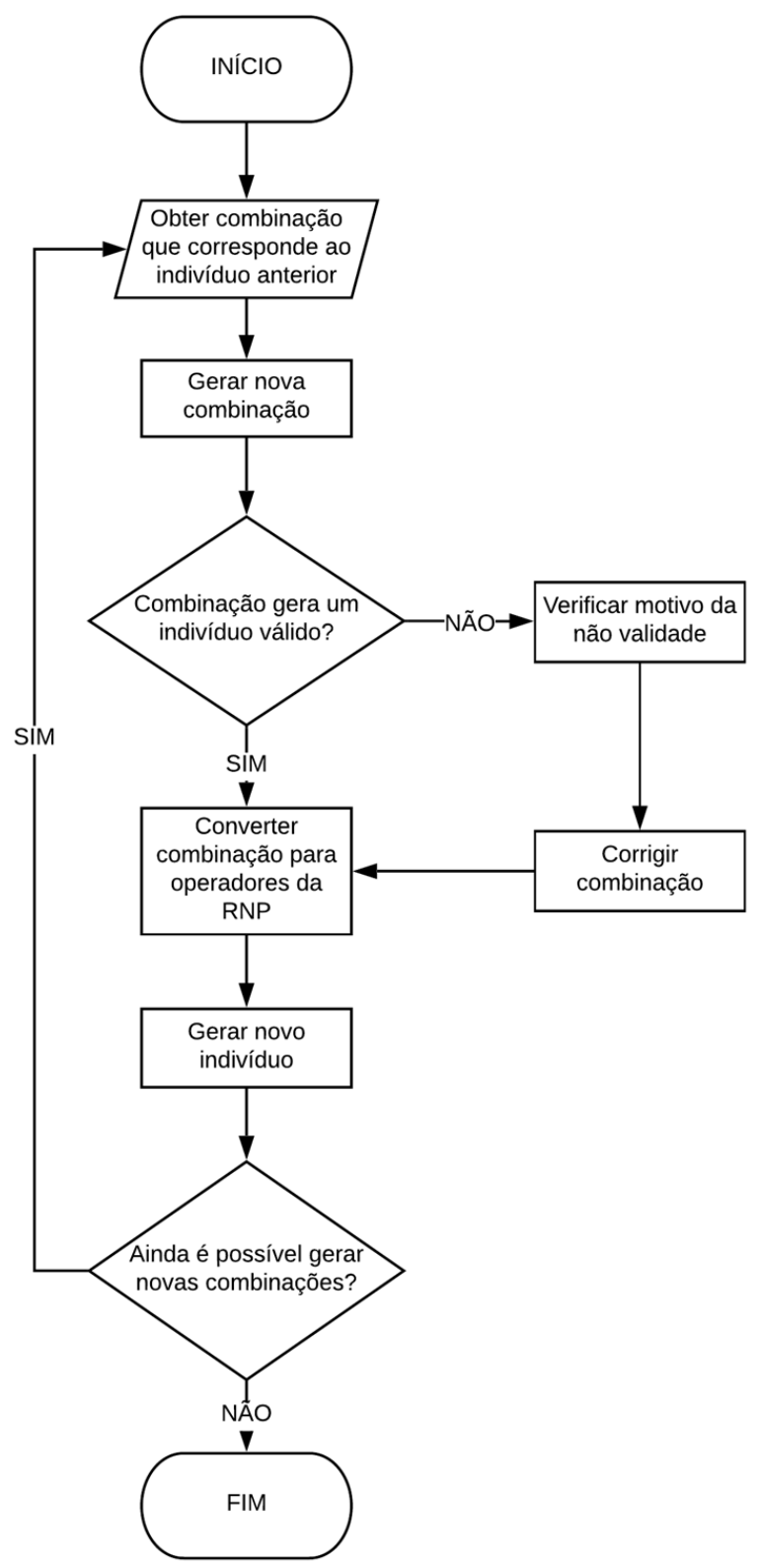

Fig. 2 Fluxograma do processo de busca local.

\subsubsection{Operador de Corte de Cargas}

Posteriormente à etapa das buscas BELM e BL, o método segue para a aplicação do processo evolutivo, conforme proposto por Marques (2018), porém com a adição de um operador de corte de cargas.

O operador de corte de cargas, chamado de Load Shedding Operator (LSO), permite a poda em uma RNP da porção energizada e o enxerto em uma RNP da porção nãoenergizada. Este operador amplia o espaço de buscas percorrido pelo algoritmo evolutivo ao permitir que seja explorada a região em que são desligados setores inicialmente não afetados pelas manobras de isolamento da falta.

Por um lado, o LSO é desejável em função de permitir a ampliação da exploração do espaço de busca. Por outro lado, é indesejável, uma vez que pode levar ao aumento de número de manobras e ENS. Dessa forma a sua aplicação deve ser controlada. Portanto, são estabelecidos alguns critérios para definir sua aplicação: somente poderão ser feitos cortes em indivíduos que sejam infactíveis e que não sejam resultado da realização de um número elevado de manobras (5, para as simulações realizadas neste trabalho), pois o alto número de manobras, somado à aplicação de cortes, leva ao crescimento na ENS total. Os cortes também são restritos aos alimentadores envolvidos na sequência de chaveamento do indivíduo selecionado, pois não há razão para se cortar qualquer setor que não corrobora com a factibilidade daquele indivíduo. $\mathrm{O}$ LSO ainda tem grande importância ao diminuir as chances de o processo evolutivo ficar preso em uma região restrita do espaço de buscas pelo fato de a BL ter a chance de popular todas as tabelas com indivíduos semelhantes (caso sejam encontrados vários indivíduos com características similares aptos a preencher as tabelas, dado que a quantidade de indivíduos gerada na BL é bem mais alta que a quantidade que pode ser armazenada nas tabelas na maioria das situações), deixando a população inicial do processo evolutivo pouco diversa.

\section{TESTES, RESULTADOS E DISCUSSÕES}

Para a avaliação do método proposto, foram simulados casos de faltas simples no SD já analisado em (Romero et al. 2016; Marques 2018), representado na Fig. 3, composto por 53 setores, 3 subestações, 6 alimentadores, 53 barras e 61 chaves manuais, com um carregamento máximo de rede de 75,2\%, queda máxima de tensão de $2,8 \%$ e carregamento máximo de subestação (um transformador) de 76,7\%. O tempo de manobra considerado para chave manual foi de $25 \mathrm{~min}$, com o tempo total estimado de $4 \mathrm{~h}$ para que a falta seja plenamente corrigida, conforme proposto por Romero et al. (2016) e Zidan e El-Saadany (2012). O limite máximo de queda de tensão considerado foi de $10 \%$. A quantidade de gerações a ser processada pelo método proposto, considerando BL e etapa evolutiva foi de 20.000 (no método de Marques (2018) as gerações são contabilizadas somente na etapa evolutiva). Todas os casos de falta simples foram simulados 50 vezes, com o método proposto e o método de Marques (2018), usando sempre o mesmo computador com processador Core i3 8100 e 16GB RAM DDR4 a $2400 \mathrm{MHz}$, rodando o sistema Ubuntu 18.04 LTS.

A avaliação do desempenho do método proposto foi realizada através da comparação entre as soluções finais encontradas ao longo de 50 simulações, considerando a diversidade do conjunto e número de vezes em que cada solução foi obtida.

Dos 14 casos de falta em que há necessidade de se executar a BL e o processo evolutivo, foram escolhidos 3 , referentes às barras 3, 4 e 11, por serem representativos das principais diferenças observadas entre os métodos.

\subsection{Falta simples na barra 3}


A ocorrência de falta na barra 3 implica no desligamento das barras 4, 5, 6, 7, 8, 26, 27 e 28, sendo este o caso com maior valor de energia não suprida em todo o sistema após a obtenção de um PRE.

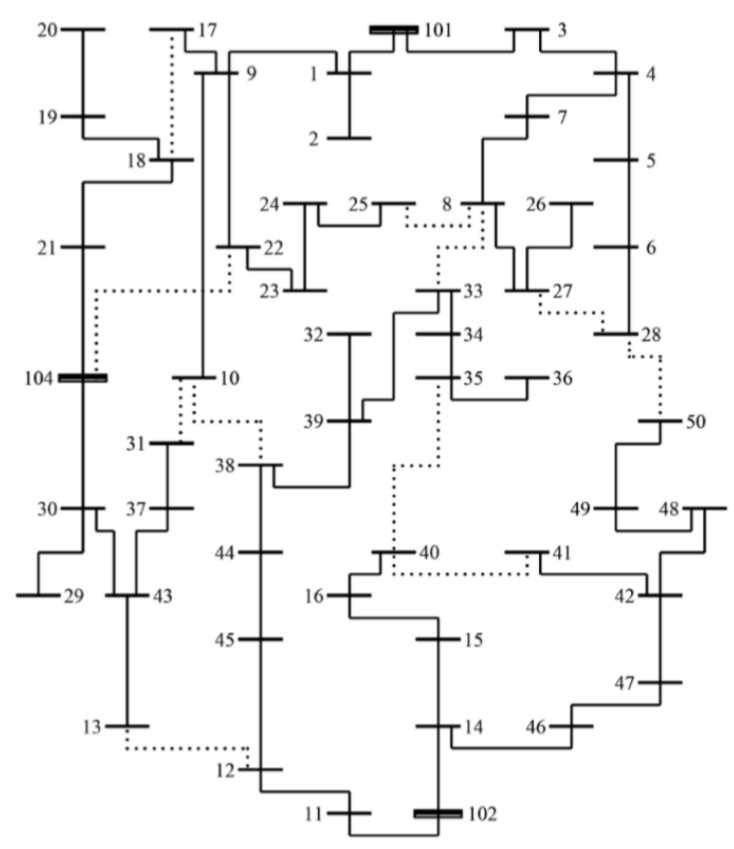

Fig. 3 Sistema de 53 barras representado no formato barrachave em sua configuração pré-falta (Romero et al. 2016).

Na Fig. 4 são mostrados os valores, para os objetivos do problema, do conjunto Pareto-Ótimo obtido pelos métodos ao final de 50 simulações de falta na barra 3 . Também são mostradas as configurações que são encontradas pelo processo de busca local, ou seja, soluções sem a realização de manobras que provocam desligamentos temporários.

Nota-se que, com exceção de um ponto em que ambos são iguais, o método proposto foi capaz de minimizar os valores de ENS, reduzindo o número manobras em chaves. Ressaltase ainda que os indivíduos que compõem a conjunto ParetoÓtimo das soluções do método proposto são todos obtidos pela BL, sendo livres de desligamentos temporários em consumidores não afetados pela falta e independentes do processo evolutivo, sendo obtidos em todas as 50 simulações.

Observa-se, ao comparar as soluções encontradas, que o método proposto, em sua configuração de menor ENS (19790,92 kWh/fase, com 7 manobras), apresenta PNS $(5128,20 \mathrm{~kW} /$ fase $)$ consideravelmente mais alta que a melhor solução em ENS (20131,65 kWh/fase, com 8 manobras e PNS de $3257,10 \mathrm{~kW} /$ fase) encontrada pelo método de Marques (2018), mostrando que o não desligamento temporário de setores saudáveis pode ser bastante benéfico para os objetivos considerados na formulação utilizada. Para fins de comparação, o método de Marques (2018) realiza 3 manobras de abertura de chaves na parte energizada, tendo, ao fim do processo, apenas 3 setores desenergizados. Já o método proposto não realiza manobras na parte energizada, tendo ao fim do processo, 5 setores desenergizados. Juntamente com o fenômeno observado no valor da PNS, trata-se de um indicativo de que os valores finais de PNS e quantidade de setores desenergizados não são necessariamente representativos do valor da ENS, sendo extremamente importante levar em conta a sequência de chaveamento.

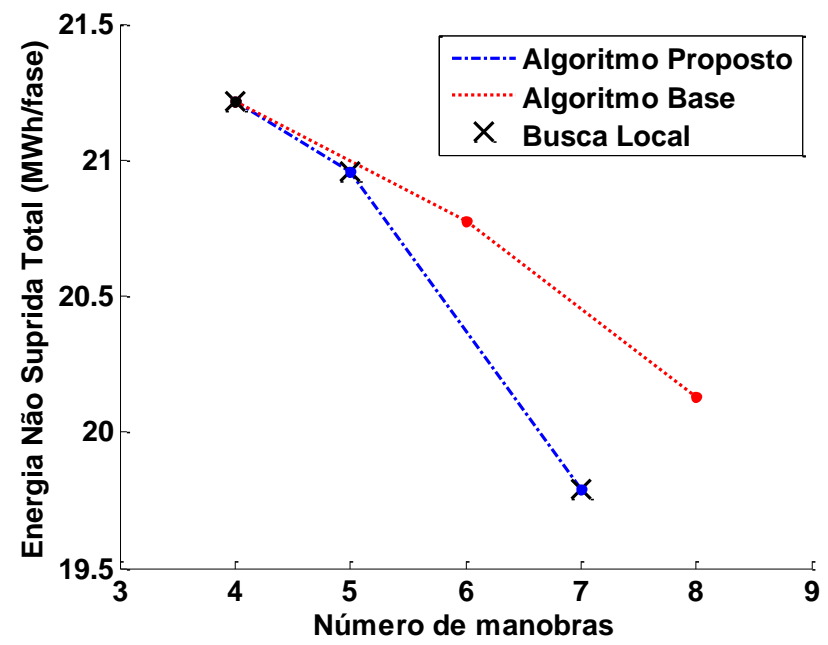

Fig. 4 Conjunto Pareto Ótimo - Falta na Barra 3.

\subsection{Falta simples na barra 4}

Novamente, todas as soluções que formam o conjunto ParetoÓtimo do método proposto foram obtidas ainda na etapa da BL. Desta vez, porém, não há nenhum ponto em comum entre as melhores soluções para os dois métodos. Ainda assim, duas das três soluções encontradas no método de Marques (2018) possuem menor PNS, se comparadas com as soluções de característica similar no método proposto. Mais uma vez, isso deixa claro as grandes vantagens de se buscar por soluções concentradas na região problema, além de mostrar novamente que não se pode garantir que uma configuração com menor PNS ao final terá também menor ENS sem conhecer os tempos de manobras e a sequência de chaveamento. Os resultados obtidos são mostrados na Fig. 5.

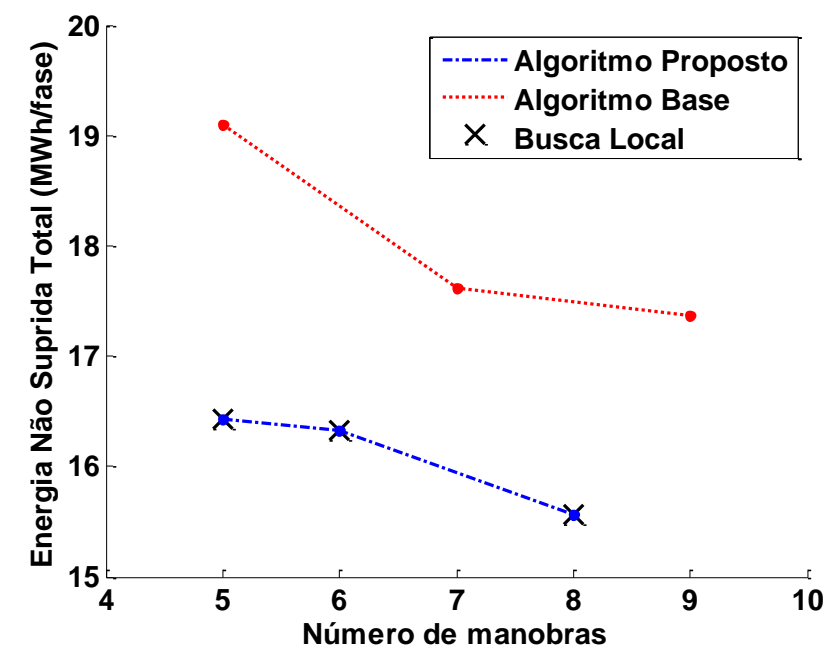

Fig. 5 Conjunto Pareto Ótimo - Falta na Barra 4. 
O caso de falta na barra 4 é o que apresenta a maior diferença nos resultados obtidos pelos métodos comparados, em virtude, especialmente, da execução da BL.

Assim como no caso anterior, como a aproximação do conjunto Pareto-Ótimo obtido pelo método proposto é composto somente por soluções obtidas pela BL, em todas as 50 simulações são obtidos os mesmos resultados.

\subsection{Falta simples na barra 11}

Neste caso, os dois métodos encontraram a mesma solução em termos de menor ENS. Esta solução não pertence ao espaço de busca percorrido pela BL, tendo, portanto, ocorrências de desligamentos temporários. Mesmo assim, a BL ainda foi capaz de encontrar duas soluções que compõem o conjunto de soluções finais, além de uma terceira solução, que mesmo dominada, apresenta uma alternativa para o operador que não deseja afetar negativamente o FEC ou quer evitar problemas por quedas temporárias de energia, conforme a Fig. 6. Neste caso especificamente, estão apresentadas todas as soluções finais obtidas ao longo das 50 simulações em conjunto no mesmo gráfico.

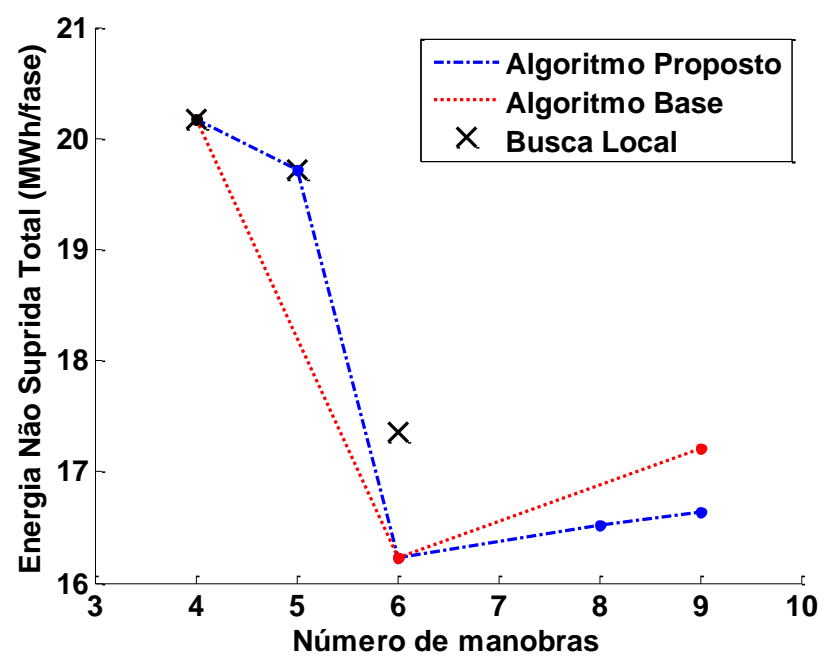

Fig. 6 Conjunto Pareto Ótimo - Falta na Barra 11.

Este caso mostra ainda que a BL e o LSO afetam positivamente a diversidade de soluções obtidas na etapa evolutiva do método, dado que foram encontradas cinco soluções para compor o conjunto Pareto-Ótimo no método proposto, ante três do método base, sem prejuízo às características dos PREs encontrados no método base. Porém, o aumento na diversidade de soluções também indica que os resultados obtidos não são constantes ao longo das 50 simulações. A solução mostrada com 4 manobras apareceu no conjunto Pareto-Ótimo nas 50 soluções, a com 5 em 47, a com 6 em 44, sendo que as demais configurações apareceram somente 2 vezes cada, indicando que o resultado obtido é consistente na região de soluções de menor número de manobras, mesmo com pequenas variações.

Por fim, acrescenta-se que o método proposto não aumentou o tempo de execução do código em nenhum dos casos. Todas as simulações ficaram dentro do intervalo de $3 \mathrm{~s}$, não representando uma parcela significativa do tempo total de restauração completa estimado para a rede, de $4 \mathrm{~h}$, e nem mesmo do tempo de $3 \mathrm{~min}$ para que se comece a contabilizar os indicadores DEC e FEC.

\section{CONCLUSÃO}

O método proposto se mostrou bastante eficiente na obtenção de soluções adequadas nos testes apresentados em um sistema de pequeno porte, sendo capaz de encontrar vários PREs com ganhos significativos nos objetivos e, em alguns casos, pode resultar em maior diversidade de soluções em comparação com o método base.

Ainda que não fosse o objetivo principal deste trabalho, os resultados obtidos permitiram uma visão mais ampla da relação entre potência não suprida e energia não suprida, mostrando que a filosofia de operação da rede pode interferir em como são vistos os resultados obtidos. Ao reduzir os desligamentos temporários, a potência não suprida se torna muito menos significativa para a energia não suprida. Deste modo, não é possível comparar diretamente métodos que lidam com a linha do tempo do problema de restabelecimento com aqueles que tem como objetivo unicamente a redução do número de consumidores desenergizados.

Vislumbram-se o desenvolvimento de trabalhos futuros para validação desta metodologia em sistemas de grande porte, onde o consumo de memória RAM do processo de busca local pode se tornar um fator limitante. Pode ser necessário a aplicação de simplificações na busca local ou a concentração em regiões promissoras para garantir a viabilidade computacional.

\section{AGRADECIMENTOS}

Os autores agradecem à Universidade de São Paulo (USP) pelo apoio. Também agradecem às agências de fomento CAPES e CNPq (processos no 134387/2018-0 e 308297/2018-0) e à Fundação de Amparo à Pesquisa do Estado de São Paulo (FAPESP) (processo 2017/23728-0) pelo apoio financeiro.

\section{REFERÊNCIAS}

Agência Nacional de Energia Elétrica - ANEEL (2018). Procedimentos de Distribuição de Energia Elétrica no Sistema Elétrico Nacional - Módulo 8. Rev. 10, Brasília, 88.

Benayoun, R., Montgolfier, J., Laritchev, O. (1971). Linear programming with multiple objective funcitions: Step method (stem). Journal Mathematical Programming, 1(1), 366-375.

Camillo, M. H.M. et al. (2016). Combining exhaustive search and multi-objective evolutionary algorithm for service restoration in large-scale distribution systems. Electric Power Systems Research, 134, 1-8.

Deb, K. et al. (2002). A fast and elitist multiobjective genetic algorithm: NSGA-II. Evolutionary Computation, IEEE Transactions on, 6(2), 182-197. 
Delbem, A. C. B., Carvalho, A. C. P. L. F., Policastro, C., Pinto, A. K. O., Garcia, A. and Honda, K. (2004). Nodedepth Encoding for Evolutionary Algorithms Applied to Network Design. Genetic and Evolutionary Computation Conference.

Marques, L. T. (2018). Restabelecimento de energia em sistemas de distribuição considerando aspectos práticos. Tese de Doutorado, Universidade de São Paulo. DOI: 10.11606/T.18.2018.tde-26072018-134924

Miu, K. N., Chiang, H-D. and Mcnulty, R. J. (2000). MultiTier Service Restoration through Network Reconfiguration and Capacitor Control for Large-Scale Radial Distribution Systems. IEEE Transactions on Power Systems, 15(3), 1001-1007.

Mohammadi, F. and Afrakhteh, H. (2012). Optimal load restoration in distribution network using intentional islanding. Journal of Electrical Engineering, 12(4), 108113.

Romero, R., Franco, J. F., Leão, F. B., Rider, M. J. and de Souza, E. S. (2016). A New Mathematical Model for the Restoration Problem in Balanced Radial Distribution Systems. IEEE Transaction on Power Systems, 31(2), 1259-1268.

Santos, A. C., Delbem, A. C. B., London, Jr., J. B. A. and Bretas, N. G. (2010). Node-Depth Encoding and MultiObjective Evolutionary Algorithm Applied to LargeScale Distribution System Reconfiguration. IEEE Transactions on Power Systems, 25(3), 1-12.

Shin, D.-J., Kim, J.-O., Kim, T.-K. and Choo, J.-B. and Singh, C. (2004). Optimal service restoration and reconfiguration of network using Genetic-Tabu algorithm. Electric Power Systems Research, Elsevier, 71(2), 145-152.

Zidan, A. and El-Saadany, E. (2012). A Cooperative Multiagent Framework for Self-Healing Mechanisms in Distribution Systems". IEEE Transactions on Smart Grid, 3(3), 1525-1539. 